\title{
Determinación de los Signos y Síntomas de los Trastornos Temporomandibulares, en Estudiantes de 13 a 18 Años de un Colegio de la Comuna de Temuco, Chile
}

\author{
Temporomandibular disorders signs and symtoms determination \\ of 13 to 18 years old students from a school in Temuco, Chile
}

"Gilda Corsini; "* Ramón Fuentes; *** Luis Bustos; **Evelyn Borie; ${ }^{* * * *}$ Andres Navarrete; ${ }^{* * * *}$ Diego Navarrete \& ${ }^{* *}$ Bruno Fulgeri.

CORSINI, G.; FUENTES, R.; BUSTOS, L.; BORIE, E.; NAVARRETE, A.; NAVARRETE, D. \& FULGERI, B. Determinación de los signos y síntomas de los trastornos temporomandibulares, en estudiantes de 13 a 18 años de un colegio de la comuna de Temuco, Chile. Int. J. Morphol., 23(4):345-352, 2005.

RESUMEN: Los trastornos temporomandibulares (TTM) han sido descritos como "término colectivo que encierra muchos problemas clínicos que comprometen la musculatura masticatoria, articulaciones y estructuras asociadas". Estudios epidemiológicos han reportado que éstos son comunes en niños y adolescentes, con prevalencias de $6 \%$ a $68 \%$ y, en población adulta el $75 \%$ presenta algún tipo de alteración funcional. Los propósitos de este estudio fueron conocer la prevalencia de signos y síntomas de TTM, en un grupo de escolares de Temuco, Chile y determinar tanto el efecto de diseño para un posterior estudio poblacional, como la estabilidad de los tests.

Es un estudio de corte transversal, con muestra no probabilística por conveniencia y consentimiento informado, de 116 alumnos del Colegio Bautista de Temuco, quienes respondieron un test sobre síntomas subjetivos de TTM, y fueron examinados clínicamente evaluando: alteraciones, limitaciones o desviación de los movimientos mandibulares, dolor a la palpación muscular y articular, ruidos y bruxismo.

El test autorreporte arrojó que: el 77.6\% mostró uno o más síntomas; el 37.9\% presentó ruido articular; 23.3\% sintió la mandíbula rígida y un 35.3\% refirió dolor de cabeza, nuca y sien. Clínicamente, el 85.3\%\% presentó uno o más signos de TTM. Un 50\% presentó ruido articular, el $8.6 \%$ alteración apertura bucal, $18.1 \%$ disminución del movimiento en lateralidad, $8.6 \%$ sensibilidad a la palpación articular y el $4.3 \%$ y $6 \%$ a la palpación de los músculos temporal y masetero, respectivamente y el $46.6 \%$ alguna manifestación de bruxismo. Al comparar estos signos con edad y sexo, sólo hubo diferencias significativas de la edad con apertura y bruxismo ( $<<0.05)$.

En conclusión, el ruido fue el signo de mayor frecuencia en esta población. Hay concordancia entre lo percibido por los adolescentes y lo observado clínicamente en cuanto a: apertura, dificultad al masticar, ruido y sensibilidad articular. En los análisis del test y retest, se encontró alta concordancia entre las respuestas. No hubo diferencias estadísticas entre la presencia de signos y síntomas respecto al sexo.

PALABRAS CLAVE: Trastornos temporomandibulares; signos y síntomas; prevalencia

\section{INTRODUCCIÓN}

Los trastornos temporomandibulares (TTM), en sî mismos, forman un grupo de problemas clínicos que comprometen la musculatura masticatoria, las articulaciones temporomandibulares (ATMs), y las estructuras asociadas, o ambas (McNeill, 1993; Okeson, 2003; Goldstein, 1999;
McNeill, 1997). Se considera que tales condiciones constituyen una subclasificación de los desórdenes musculoesqueletales y han sido identificados como una causa importante del dolor no dentario en la región orofacial (McNeill, 1993; Dimitroulis, 1998; Greene, 1995).

\footnotetext{
Departamento de Odontología Integral, y CIGES, Facultad de Medicina, Universidad de La Frontera, Temuco, Chile.

** Departamento de Odontología Integral, Facultad de Medicina, Universidad de La Frontera, Temuco, Chile.

*** CIGES y Departamento de Salud Pública, Facultad de Medicina, Universidad de La Frontera, Temuco, Chile.

***** Carrera de Medicina, Facultad de Medicina, Universidad de La Frontera, Temuco, Chile.

Proyecto N EP-120238. Dirección de Investigación y Desarrollo, Universidad de La Frontera, Chile.
} 
Los TTM se caracterizan clínicamente por dolor en músculos de la masticación, en el área preauricular y/o en la ATM, usualmente agravado por la manipulación y la alteración de los movimientos mandibulares (limitado rango de movimiento, movimiento asimétricos), y/o ruidos articulares como popping, crepitación y clicking (McNeill, 1993; List et al., 1999; Wijer et al., 1995; Conti et al.,1996), que en los niños usualmente son leves y en los adolescentes alcanzan porcentajes del 75.8\% (como signo único) (Barone et al., 1997), no existiendo evidencias científicas de que progresen a condiciones más severas en la edad adulta (Könönen et al., 1996). El dolor se ha descrito ser de mayor predominio en las mujeres que en los hombres (DeBont et al., 1997; Goldstein) y en la pubertad y edad media, más que en ancianos y niños pequeños.

Estudios epidemiológicos de TTM han revelado una alta prevalencia y una gran variabilidad en sus tasas (Egermark-Eriksson et al., 1997; Wahlund et al., 1998) de un $6 \%$ a un $68 \%$, en poblaciones de adolescentes y niños (List et al.; Krogstad et al., 1992) y del 20\% al 70\%, en la población general (Magnusson et al., 1985)

Estudios transversales y longitudinales de poblaciones específicas muestran que, aproximadamente, el $70 \%$ al $75 \%$ de la población adulta tiene, a lo menos, un signo de TTM (McNeill, 1993; Dimitroulis) y de éstos, uno de cada 4 sujetos, con signo de disfunción estará consciente o lo informará. Según Salonen et al. (1990), la prevalencia de síntomas fluctúa de un 16\% a un 50\% y los signos de un 33\% a un $86 \%$.

Estas considerables variaciones en la prevalencia de signos y síntomas de TTM se deben, probablemente, a la falta de métodos de examen estandarizados válidos y confiables, que hagan posible la comparación entre los diferentes estudios, a deficiencias metodológicas y a falta de definicion y caracterización de los TTM, más que a reales diferencias de las muestras en estudio (List et al.; Magnusson et al.; DeBont et al.; McNeill, 1997; LeReche, 1997), causas todas éstas también del fracaso, perpetuación de los tratamientos y serias complicaciones psicosociales en pacientes aquejados por estos problemas.

Independientes de estas variaciones, los signos y síntomas de los TTM son comunes en población de adultos, niños y adolescentes, tanto en población con problemas como en la población de sujetos sanos; por ejemplo, el clicking, se presenta en el 33\% de los sujetos de población sana (DeBont; Goldstein), lo que significa que los TTM no constituyen una enfermedad simple y que pueden llevar a confusión si no se saben diagnosticar y manejar estas condiciones.
La etiología de los signos y síntomas de los TTM, tópico que aún permanece sin aclarar, ha generado controversias y conflictos y múltiples hipótesis que pretenden explicar su aparición (Greene; Thurk, 1998; Kuttila et al. 1998). Se acepta que es multifactorial, con un fuerte componente biosicosocial (Goldstein; McNeill 1997). Las razones para esto, son complejas e, indudablemente, son el resultado de muchos factores, incluyendo la historia natural de la enfermedad misma, como también la existencia de una sobre dependencia de los reportes no científicos pero aceptados como un "hecho probado" de sucesos clínicos, junto con la necesidad de dar solución a las quejas, la falta de estudios epidemiológicos y de aplicación de la evidencia científica.

Es por ello que hoy en día debemos preocuparnos en desarrollar investigación confiable, con protocolos claros y rigurosos, que respalden la evidencia clínica existente con respecto a este tema.

Los objetivos del presente estudio piloto, han sido determinar la prevalencia de signos y síntomas y factores asociados de TTM, en adolescentes de 13-18 años, establecer el efecto de diseño por curso para el cálculo del tamaño muestral para estudio poblacional y ver en terreno el comportamiento y estabilidad de test de autorreporte y test de screening.

\section{MATERIAL Y MÉTODO}

Corresponde a un estudio de corte transversal, realizado en una muestra no probabilística por conveniencia, en 116 alumnos de 13 a 18 años, de $7^{\circ}$ año de Enseñanza Básica a $4^{\circ}$ año de Enseñanza Media del Colegio Bautista de Temuco, Chile, seleccionando un curso por cada nivel, según disponibilidad horaria.

A todos los sujetos que aceptaron participar (consentimiento firmado), se les entregó un cuestionario de autorreporte y luego se les realizó un examen de screenig clínico, practicado por personal capacitado y adiestrado previamente. Los instrumentos de recolección de datos fueron los test de screenig de autorreporte y clínico, recomendados por la American Academy of Oralfacial Pain (McNeill 1993) y en las modificaciones realizadas por Kindernecht (1997).

El test de autorreporte, (Tabla I) contiene 11 preguntas, a las cuales deben responder "si" o "no", referentes a signos subjetivos, detectados por ellos mismos, en relación a su ATM, oclusión, musculatura masticatoria, movimientos mandibulares, bruxismo, golpes o accidentes y tratamientos anteriores. Los alumnos recibieron claras instrucciones 
de cómo llenar la pauta, permaneciendo en todo momento un evaluador con ellos para responder las dudas que se presentaran.

El test de screening clínico (Tabla II ), evalúa en 116 alumnos signos y síntomas de TTM, tales como; a) alteraciones, limitaciones y/o desviación de los movimientos mandibulares en apertura y cierre y laterotrusión derecha e izquierda, medidos todos con compás seco y regla, anotando también cualquier falta de coordinación de los movimientos. La apertura máxima (libre de dolor), se determinó desde los bordes incisales de los incisivos centrales superiores e inferiores, registrándose las medidas de over-bite y over-yet; b) Dolor a la palpación de los músculos masetero y temporal. La evaluación se realizó usando el método del dolor a la presión con la yema del dedo, que consistió en aplicar una presión estándar a lo largo del músculo con la extremidad del dedo del índice, igual a la presión aplicada en la mano entre los dedos pulgares e índice y que el paciente hubiese manifestado sentir ligero dolor. El examinador fue entrenado para aplicar esta misma presión en todos los músculos. c) Dolor a la palpación articular, evaluación realizada intrameatalmente, a través del meato auditivo externo usando el dedo mínimo para ejercer presión hacia adelante, contra la cara posterior del proceso condilar, con los dientes en posición intercuspídea, y palpación parauricular, determinada aplicando ligera presión con el dedo índice sobre el polo lateral del processo condilar apenas anterior al tragus. Para facilitar la identificación del polo lateral, se pidió a los pacientes abrir ligeramente su boca. d) Los ruidos (clicking y crepitación), fueron determinados aplican- do ligera presión con el dedo índice sobre la ATM, seguido por auscultación con un estetoscopio para los ruidos y crepitaciones finas. e) Los signos de parafunción, como bruxismo, se determinaron según facetas de desgaste presentes en las piezas dentarias. Mediante estos breves exámenes no se pretendió diagnosticar los problemas de desarreglos internos de la articulación, ni menos dar indicaciones para su tratamiento, sólo indicar las áreas donde se presentan problemas (síntomas de TTM). Los exámenes fueron realizados por profesionales Odontólogos con experiencia en TTM y especialistas en Ortodoncia y alumnos de quinto año de la Carrera de Odontología, quienes fueron preparados para realizar y anotar correctamente los resultados en fichas de recolección de datos preparadas para ese fin y basados en los protocolos propuestos por la AAOP.

El test de autorreporte, se pasó por segunda vez a los 6 meses, en 45 de los 116 estudiantes (test-retest). La pérdida de sujetos se debió a su egreso del Colegio, o no presentación el día en que fue aplicado por segunda vez este test.

Análisis estadístico: Se realizó un análisis descriptivo de las variables bajo estudio, a través de tablas estadísticas y medidas de resumen. La prueba estadística para evaluar el test retest fue la prueba exacta de Mc Nemar. La comparación de las variables cualitativas, mediante. Las pruebas de Chi-cuadrado o Prueba exacta de Fisher, según corresponda. Las variables cuantitativas se compararon con la prueba t de Student para varianzas iguales y diferentes. El nivel de significación utilizado fue de $5 \%$.

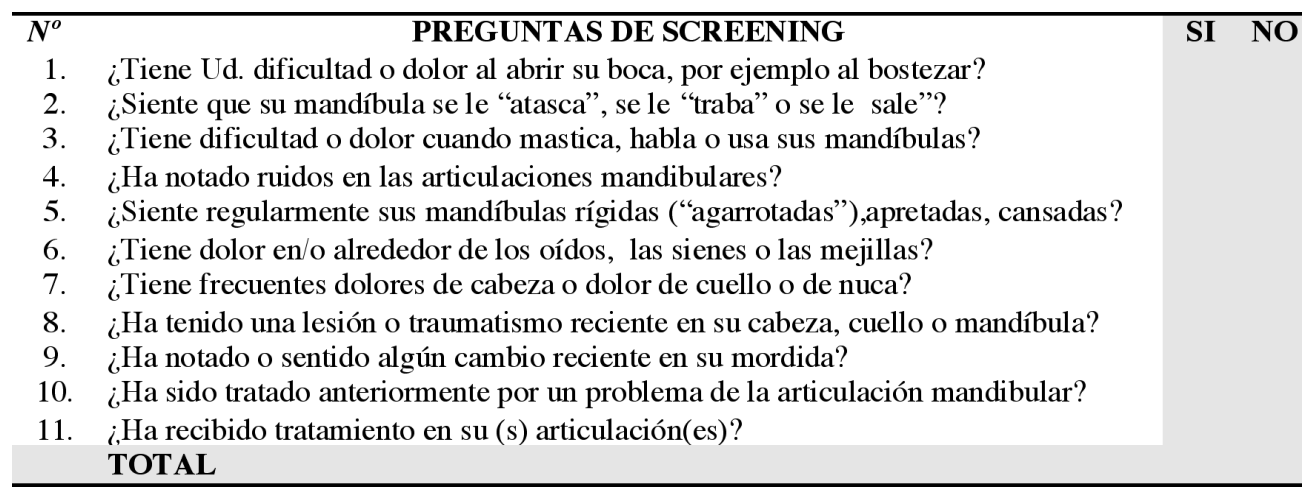

Tabla I. Cuestionario de Screening (Recomendado para los DMT, por la American Academy of Oralfacial Pain, en 1993).

\footnotetext{
- Medir el rango de movimiento de la mandíbula en apertura y laterotrusión derecha e izquierda.

- Anotar cualquier falta de coordinación en los movimientos.

- Palpar en busca de sensibilidad de la ATM preauricular o intrameatal.

- Auscultar y/o palpar los sonidos de la ATM (es decir, clicking o crepitación).

- Palpar la sensibilidad en los músculos masetero y temporal.

- Observar el desgaste oclusal excesivo, excesiva movilidad de los dientes, la altura (ridging), de la mucosa bucal o improntas laterales de la lengua.

- Inspeccionar la simetría y alineación de la cara, mandíbulas y arcos dentales.

Tabla II. Procedimientos de Examen Clínico de Screening (Recomendado por la American Academy of Oralfacial Pain, en 1993).
} 


\section{RESULTADOS}

Análisis del Test Screaning de Autorreporte. En una muestra de 116 estudiantes, de los cuales $50.9 \%$ fueron mujeres, cuya edad promedio fue $15 \pm 1.6$ años, con un rango de edad de 13 a 18 años, se encontró que el síntoma percibido con mayor frecuencia fue el ruido articular, 37.9\%. Sentir la mandíbula rígida en las mañanas fue relatado por el $23.3 \%$ de los encuestados. Tener problemas al abrir la boca $8.6 \%$, que se traba la mandíbula $19 \%$, dolor de cabeza, sienes y nuca $35.3 \%$ y dolor alrededor de los oídos $12.1 \%$. Aquellos que informaron haber sufrido un traumatismo como golpe de látigo por accidente o golpe directo a la mandíbula fue el $4.3 \%$ y aquellos que habían notado en el último tiempo que su mordida había cambiado de posición o que ya no mordía de la misma manera, el $10.3 \%$. En términos generales, el $77.6 \%$ de los adolescentes informaron presentar, a lo menos, un síntoma subjetivo de TTM (Tablas III y IV).

Al comparar presencia de síntomas con la edad, no se encontraron diferencias estadísticamente significativas (Tabla V). Su distribución según sexo resultó estadísticamente significativa en los siguientes síntomas: mandíbula rígida, dolor alrededor de los oídos, dolor de cabeza-nucasien $(\mathrm{p}<0.05)($ Tabla VI)

Análisis del test de Screening Clínico. El test de screening arrojó los siguientes resultados: el promedio de la apertura interincisal fue de $46.16 \mathrm{~mm}$ (DE $6.30 \mathrm{~mm}$ ), con un rango entre $28 \mathrm{~mm}$ y $68 \mathrm{~mm}$; el promedio de la apertura (sumado el overbite) fue de $49.53 \mathrm{~mm}$ (DE $6.48 \mathrm{~mm}$ ), con un rango de $31 \mathrm{~mm}$ y $68 \mathrm{~mm}$, siendo esta diferencia de la medición de la apertura bucal, con o sin over-bite, altamente significativas $(\mathrm{p}=0.0001)$. El promedio del movimiento en lateralidad derecha fue de $8.9 \mathrm{~mm}(\mathrm{DE} 2.15 \mathrm{~mm})$ y en lateralidad izquierda fue $8.5 \mathrm{~mm}$ (DE $2.16 \mathrm{~mm}$ ), el rango del movimiento fluctuó para ambos entre $3 \mathrm{~mm}$ y $14 \mathrm{~mm}$, existiendo entre ambas magnitudes de desplazamiento una diferencia significativa $(\mathrm{p}=0.013)$. El $8.6 \%$ presentó alteración en la apertura bucal, el 50\% ruido articular (tipo clicking o crepitación), siendo el signo de mayor prevalencia en esta muestra. En el $18.1 \%$ se encontró una disminución del movimiento en lateralidad (derecha o izquierda). La sensibilidad a la palpación articular (intrameatal y parauricular), se presentó en el $8.6 \%$. El $4.3 \%$ y $6 \%$ presentó sensibilidad a la palpación de los músculos temporales y maseteros, respectivamente; el $46.6 \%$ presentó alguna manifestación clínica de bruxismo y el $22.4 \%$, sólo bruxofacetas. La desviación mandibular en "S" durante la apertura, fue de un $17.2 \%$ (Tabla VII). El $85.3 \%$ de la muestra examinada presentó, a lo menos, un signo de TTM al examen clínico (Tabla VIII).
Al comparar estos signos con edad y sexo, sólo hubo diferencias significativas de la edad con alteración en la apertura y bruxismo $(\mathrm{p}<0.05)$ (Tabla IX). Su distribución según sexo, resultó no ser estadísticamente significativa con ningún signo ( $p>0.05)$ (Tabla $X)$.

Análisis del Test de Screening de Autorreporte versus Test de Screening Clínico. En el grupo estudiado se compararon signos y síntomas mediante la prueba de McNemar, se determinó concordancia entre las respuestas del test de autoreporte y los signos encontrados en el test clínico, en aquellas referentes a: problemas al abrir la boca $(\mathrm{p}=0.458)$, dificultad al abrir la boca $(\mathrm{p}=0.1153)$, ruido articular $(\mathrm{p}=0.0595)$ y sensibilidad a la palpación articular $(\mathrm{p}=0.5034)$.

Test- retest:Los resultados del test-retest, en una muestra de 45 estudiantes, indican una alta concordancia en las respuestas relacionadas con la presencia de signos y síntomas (Mac Nemar, $\mathrm{p}>0.05$ ).

Tabla III. Frecuencia de signos y síntomas de trastornos temporomandibulares en 116 alumnos de Enseñanza Media del colegio Bautista de Temuco, Chile (test de screening de autorreporte).

\begin{tabular}{lcr}
\hline Sígnos y síntomas & $\mathrm{N}^{\mathrm{o}}$ & \multicolumn{1}{c}{$\%$} \\
\hline Problemas al abrir la boca & 10 & 8,6 \\
Se traba la mandíbula & 22 & 19,0 \\
Problemas al masticar & 10 & 8,6 \\
Ruido articular & 44 & 37,9 \\
Mandíbula rígida & 27 & 23,3 \\
Dolor alrededor de oídos & 14 & 12,1 \\
Dolor cabeza, nuca y sien & 41 & 35,3 \\
Traumatismos & 5 & 4,3 \\
Cambio en la mordida & 12 & 10,3 \\
Tratamiento articular & 3 & 2,6 \\
\hline
\end{tabular}

Tabla IV. Número de respuestas positivas a la presencia de síntomas autoreportados de trastornos temporomandibulares en 116 alumnos de Enseñanza Media del Colegio Bautista de Temuco, Chile.

\begin{tabular}{ccr}
\hline $\begin{array}{c}\mathbf{N}^{\mathbf{0}} \text { de síntomas } \\
\text { autorreportados }\end{array}$ & $\mathbf{N}^{\mathbf{0}}$ & $\%$ \\
\hline 0 & 26 & 22,4 \\
1 & 35 & 30,2 \\
2 & 25 & 21,5 \\
3 & 19 & 16,4 \\
4 & 9 & 7,8 \\
5 & 2 & 1,7 \\
\hline Prevalencia & \multicolumn{2}{c}{$77,6 \%$} \\
Promedio \pm Desviación estándar & \multicolumn{2}{c}{$1,62 \pm 1,30$} \\
\hline
\end{tabular}


Tabla V. Frecuencia de los signos y síntomas de trastornos temporomandibulares en 116 alumnos de Enseñanza Media del Colegio Bautista de Temuco, Chile según edad (Test de screening de autorreporte).

\begin{tabular}{|c|c|c|c|c|c|c|c|c|c|c|c|c|c|c|c|}
\hline \multirow{3}{*}{ Signos y síntomas } & \multicolumn{15}{|c|}{ Edad } \\
\hline & \multicolumn{2}{|c|}{12} & \multicolumn{2}{|c|}{13} & \multicolumn{2}{|c|}{14} & \multicolumn{2}{|c|}{15} & \multicolumn{2}{|c|}{16} & \multicolumn{2}{|c|}{17} & \multicolumn{2}{|c|}{18} & \multirow[t]{2}{*}{$\mathrm{p}$} \\
\hline & $\mathrm{N}$ & $\%$ & $\mathrm{~N}$ & $\%$ & $\mathrm{~N}$ & $\%$ & $\mathrm{~N}$ & $\%$ & $\mathrm{~N}$ & $\%$ & $\mathrm{~N}$ & $\%$ & $\mathrm{~N}$ & $\%$ & \\
\hline Problemas al abrir la boca & 0 & 0,0 & 1 & 7,7 & 3 & 13,6 & 0 & 0,0 & 1 & 5,3 & 4 & 18,4 & 1 & 11,1 & 0.164 \\
\hline Se traba la mandíbula & 2 & 15.4 & 1 & 7,7 & 5 & 22,7 & 4 & 18,2 & 2 & 10,5 & 5 & 27,8 & 3 & 33,3 & 0.640 \\
\hline Problemas al masticar & 0 & 0,0 & 3 & 23,1 & 3 & 13,6 & 2 & 9,1 & 0 & 0,0 & 1 & 5,6 & 1 & 11,1 & 0.258 \\
\hline Ruido articular & 3 & 23,1 & 3 & 23,1 & 6 & 27,3 & 9 & 40,9 & 11 & 57,9 & 6 & 33,3 & 6 & 66,7 & 0.137 \\
\hline Mandíbula rígida & 3 & 23,1 & 3 & 23,1 & 4 & 18,2 & 7 & 31,8 & 6 & 31,6 & 2 & 11,1 & 2 & 22,2 & 0.755 \\
\hline Dolor alrededor de oídos & 2 & 15,4 & 0 & 0,0 & 4 & 18,2 & 4 & 18,2 & 2 & 10,5 & 2 & 11,1 & 0 & 0,0 & 0.642 \\
\hline Dolor cabeza, nuca y sien & 3 & 23,1 & 5 & 38,5 & 4 & 18,2 & 11 & 50,0 & 7 & 36,8 & 6 & 33,3 & 5 & 55,6 & 0.278 \\
\hline Traumatismos & 0 & 0,0 & 2 & 15,4 & 1 & 4,6 & 1 & 4,6 & 0 & 0,0 & 1 & 5,6 & 0 & 0,0 & 0.559 \\
\hline Cambio en la mordida & 0 & 0,0 & 0 & 0,0 & 1 & 4,6 & 2 & 9,1 & 2 & 10,5 & 4 & 22,2 & 3 & 33,3 & 0.081 \\
\hline Tratamiento articular & 0 & 0,0 & 1 & 7,7 & 0 & 0,0 & 0 & 0,0 & 1 & 15,3 & 0 & 0,0 & 1 & 11,1 & 0.184 \\
\hline
\end{tabular}

Prueba exacta de Fischer

Tabla VI. Distribución de la frecuencia de signos y síntomas de trastornos temporomandibulares en 116 alumnos de enseñanza media del colegio Bautista de Temuco, Chile según género (Test de screening autorreporte).

\begin{tabular}{|c|c|c|c|c|c|}
\hline \multirow[b]{3}{*}{ Signos y síntomas } & \multicolumn{5}{|c|}{ Test autorreporte } \\
\hline & \multicolumn{2}{|c|}{ Mujer } & \multicolumn{2}{|c|}{ Hombre } & \multirow[b]{2}{*}{$\mathrm{p}$} \\
\hline & $\mathrm{N}^{\circ}$ & $\%$ & $\mathrm{~N}^{\circ}$ & $\%$ & \\
\hline Problemas al abrir la boca & 6 & 10,2 & 4 & 7,0 & 0.743 \\
\hline Se traba la mandíbula & 15 & 25,4 & 7 & 12,3 & 0.07 \\
\hline Problemas al masticar & 6 & 10,2 & 4 & 7,0 & 0.545 \\
\hline Ruido articular & 21 & 35,6 & 23 & 40,4 & 0.598 \\
\hline Mandíbula rígida & 19 & 32,2 & 8 & 14,0 & 0.021 \\
\hline Dolor alrededor de oídos & 12 & 20,3 & 2 & 3,5 & 0.008 \\
\hline Dolor cabeza, nuca y sien & 26 & 44,1 & 15 & 26,3 & 0.046 \\
\hline Traumatismos & 2 & 3,4 & 3 & 5,3 & 0.677 \\
\hline Cambio en la mordida & 8 & 13,6 & 4 & 7,0 & 0.362 \\
\hline Tratamiento articular & 3 & 5,1 & 0 & 0,0 & 0.244 \\
\hline
\end{tabular}

Prueba exacta de Fischer

Tabla VII Frecuencia de signos y síntomas de trastornostemporomandibulares en 116 alumnos de Enseñanza Media del Colegio Bautista de Temuco, Chile. (Test de screening clínico).

\begin{tabular}{lrr}
\hline Signos y síntomas & $\mathrm{N}^{\circ}$ & \multicolumn{1}{c}{$\%$} \\
\hline Apertura & 10 & 8,6 \\
Ruido & 58 & 50,0 \\
Movimiento de lateralidad & 21 & 18,1 \\
Sensibilidad articular & 10 & 8,6 \\
Sensibilidad músculo Masetero & 7 & 6,0 \\
Sensibilidad músculo Temporal & 5 & 4,3 \\
Desviación mandibular en S & 20 & 17,2 \\
Bruxismo & 54 & 46,6 \\
\hline
\end{tabular}

Tabla VIII. Número de respuestas positivas a la presencia de síntomas detectados de trastornos temporomandibulares en 116 alumnos de Enseñanza Media del Colegio Bautista de Temuco, Chile. (Test de screening clínico).

\begin{tabular}{ccc}
\hline $\mathrm{N}^{\circ}$ de signos detectados & $\mathrm{N}^{\circ}$ & $\%$ \\
\hline 0 & 17 & 14,7 \\
1 & 46 & 39,7 \\
2 & 30 & 25,9 \\
3 & 15 & 12,9 \\
4 & 6 & 5,2 \\
5 & 2 & 1,7 \\
\cline { 1 - 2 } Prevalencia & $85,3 \%$ & \\
\cline { 1 - 2 } Promedio \pm Desviación estándar & $1,59 \pm 1,15$ & \\
\hline
\end{tabular}


CORSINI, G.; FUENTES, R.; BUSTOS, L.; BORIE, E.; NAVARRETE, A.; NAVARRETE, D. \& FULGERI, B.

Tabla IX. Distribución de la presencia o ausencia de signos y síntomas de trastornos temporomandibulares en 116 alumnos de Enseñanza Media del Colegio Bautista de Temuco, Chile según edad. (Test de screening clínico).

\begin{tabular}{|c|c|c|c|c|c|c|c|c|c|c|c|c|c|c|c|}
\hline \multirow{3}{*}{ Signos y síntomas } & \multicolumn{15}{|c|}{ Edad } \\
\hline & \multicolumn{2}{|c|}{12} & \multicolumn{2}{|c|}{13} & \multicolumn{2}{|c|}{14} & \multicolumn{2}{|c|}{15} & \multicolumn{2}{|c|}{16} & \multicolumn{2}{|c|}{17} & \multicolumn{2}{|c|}{18} & \multirow{2}{*}{$\mathbf{P}$} \\
\hline & $\mathbf{N}$ & $\%$ & $\mathbf{N}$ & $\%$ & $\mathbf{N}$ & $\%$ & $\mathbf{N}$ & $\%$ & $\mathbf{N}$ & $\%$ & $\mathbf{N}$ & $\%$ & $\mathbf{N}$ & $\%$ & \\
\hline Apertura & 0 & 0,0 & 0 & 0,0 & 0 & 0,0 & 3 & 13,6 & 1 & 5,3 & 5 & 27,8 & 1 & 11,1 & 0.029 \\
\hline Ruido & 4 & 30,8 & 4 & 30,8 & 11 & 50,0 & 12 & 54,6 & 11 & 57,9 & 12 & 66,7 & 4 & 44,4 & 0.370 \\
\hline Movimiento de lateralidad & 2 & 15,4 & 2 & 15,4 & 2 & 9,1 & 5 & 22,8 & 4 & 21,1 & 2 & 11,1 & 4 & 44,4 & 0.416 \\
\hline Sensibilidad ar ticular & 0 & 0,0 & 1 & 7,7 & 2 & 9,1 & 2 & 9,1 & 2 & 10,5 & 1 & 5,6 & 2 & 22,2 & 0.753 \\
\hline Sensibilidad m. masetero & 0 & 0,0 & 0 & 0,0 & 1 & 4,6 & 1 & 4,6 & 2 & 10,5 & 1 & 5,6 & 2 & 22,2 & 0.442 \\
\hline Sensibilidad m. temporal & 0 & 0,0 & 0 & 0,0 & 0 & 0,0 & 1 & 4,6 & 0 & 0,0 & 2 & 11,1 & 2 & 22,2 & 0.054 \\
\hline Desviación mandibular en $\mathbf{S}$ & 2 & 15,4 & 1 & 7,7 & 2 & 9,1 & 2 & 9.1 & 3 & 15,8 & 5 & 27,8 & 5 & 55,6 & 0.063 \\
\hline Br uxismo & 1 & 7,7 & 4 & 30,8 & 10 & 45,5 & 10 & 45,5 & 11 & 57,9 & 11 & 61,1 & 7 & 77,8 & 0.014 \\
\hline
\end{tabular}

Prueba exacta de Fischer.

Tabla X. Distribución de signos y síntomas de trastornos temporomandibulares en 116 alumnos de Enseñanza Media del Colegio Bautista de Temuco, Chile según género (Test de screening clínico).

\begin{tabular}{lrrrrr}
\hline \multirow{2}{*}{ Signos y síntomas } & \multicolumn{4}{c}{ Examen clínico } \\
\cline { 2 - 5 } & \multicolumn{3}{c}{ Mujer } & \multicolumn{3}{c}{ Hombre } & \multirow{2}{*}{$\mathrm{p}$} \\
\cline { 2 - 5 } & $\mathrm{N}^{\mathrm{y}}$ & 6,8 & 6 & 10,5 & 0.525 \\
Apertura & 4 & 52,5 & 27 & 47,4 & 0.710 \\
Ruido & 31 & 22,0 & 8 & 14,0 & 0.263 \\
Movimiento de lateralidad & 13 & 13,6 & 2 & 3,5 & $0.095^{*}$ \\
Sensibilidad articular & 8 & 10,2 & 1 & 1,8 & $0.114^{*}$ \\
Sensibilidad músculo masetero & 6 & 8,47 & 0 & 0,0 & $0.057^{*}$ \\
Sensibilidad músculo temporal & 5 & 22,0 & 7 & 12,3 & 0.164 \\
Desviación mandibular en S & 13 & 44,1 & 28 & 49,1 & 0.710 \\
Bruxismo & 26 & & & & \\
\hline
\end{tabular}

Prueba exacta de Fischer.

\section{DISCUSIÓN}

En un estudio longitudinal de 120 niños de 6 a 15 años, Magnusson et al. concluyeron que el $66 \%$ de los niños de 11 a 15 años tenían signos clínicos de TTM leves, siendo los casos severos muy raros, además, el $62 \%$ de los niños de 11 años y el 66\% de los de 15 años tenían síntomas subjetivos ocasionales. Autores como EgermackEriksson et al., en su estudio longitudinal por 5 años de 240 niños entre 7 y 15 años concluyeron que los signos y síntomas de TTM aumentaban ligeramente en frecuencia y en severidad, pero en la mayoría de los casos, se juzgaron leves. Lo mismo habrían comprobado Wänman \& Agerberg, Mcneill en 1993 y apoyados por Barone et al., quienes además añaden que estos signos y síntomas son considerados comunes en todos los grupos de edades y sin diferencia de sexo, lo que es coincidente con los resultados de este estudio, donde tampoco se encontró, al examen clínico, una asociación evidente con sexo y edad, excepto en apertura y bruxismo. Por otro lado, en un estudio de adolescentes suecos de 12 a18 años List et al. concluyeron que la prevalencia de dolor, como cefaleas y dolor miofascial aumenta con la edad y es más frecuente en mujeres que hombres, lo que concide con nuestros resultados del test de autorreporte, donde hubo diferencias significativas entre mujeres (44.1\%) y hombres (26.3\%), en relación a dolor de cabeza, nuca y sien ( $\mathrm{p}=0.046)$.

En nuestro estudio se observó una prevalencia del ruido articular (tipo click) de un $50 \%$ como signo (Screening clínico) y de $37.9 \%$ como síntoma (Autorreporte), no existiendo diferencias significativas por género o edad. Nuestros valores son más elevados que el reportado por Könönen et al. quienes reportaron un $11 \%$ de prevalencia, pero similares a los reportes de Goldstein, quien informó de una prevalencia de clicking en un 33\% de los sujetos sanos. El tipo de ruido (cliking) encontrado, es coincidente con lo manifestado por Egermack-Eriksson 
et al.; Barone et al., en cuyos reportes aparece el cliking como el más común de la población. Determinar la presencia de ruido articular es de importancia por cuanto no se tiene claridad sobre su significado, pues como plantean Könönen et al., no se sabe si el cliking es una condición inofensiva o si son patognomónicos del daño interno progresivo de la ATM.

Estudios longitudinales en poblaciones, muestran que entre el 60 y $70 \%$ de la población tienen, a lo menos, un signo de TTM, (McNeill, 1993; Dimitroulis; Salonen et al.), con un rango que fluctúa entre un $33 \%$ a $86 \%$ y los síntomas entre un 16 y 50\%. Sin embargo, en nuestro estudio el porcentaje de adolescentes que presentó a lo menos un síntoma, fue de $77.6 \%$, ligeramente, superior a los promedios reportados y el $85.3 \%$ presentó a lo menos un signo clínico y no hubo una diferencia estadísticamente significativa por género, lo cual está en concordancia con los promedios antes establecidos.

Actualmente, se está de acuerdo en que la etiología de los TTM es multifactorial, donde se combinan los factores físicos, psicosociales (ansiedad, depresión y estrés), algunos de ellos difíciles de entender, medir o evaluar. (Greene; Thurk; Kuttila et al.), además, se ha propuesto que hábitos parafuncionales, como bruxismo, serían fac- tores desencadenante de TTM., Wijer et al. aseveraron que no habría una asociación clara y estadísticamente significativa. Barone et al. determinaron que, entre el $5 \%$ y el $90 \%$ de la población, puede verse afectada, siendo más común en los hombres y aumentaría con la edad, lo cual es concidente con lo encontrado en este estudio, donde la prevalencia del bruxismo fue de $46.6 \%$ con una diferencia significativa con la edad. También es un acuerdo, que los signos y síntomas de TTM presentan una alta prevalencia y variabilidad en sus tazas, las que van del 6 al $68 \%$ en la población de adolescentes y niños (List et al.), de un 20 a $75 \%$ en adultos (con rangos del $2 \%$ al $97 \%$ ). Wahlund et al., en un estudio de población sueca de 12 a 18 años, en 1998, subrayaron que los métodos de diagnóstico estandarizados y los criterios de diagnóstico válidos y confiables, son absolutamente necesarios para definir e identificar los subtipos de TTM. Afirmación avalada por la mayoría de los investigadores (McNeill, 1997; Le Reche) de la actualidad y por quienes realizaron este estudio.

AGRADECIMIENTOS: Nuestros sinceros agradecimientos a los alumnos, profesores y autoridades del Colegio Bautista de Temuco, Chile y, en forma especial a los alumnos de las Carreras de Odontología y Medicina de la Universidad de La Frontera, Chile, por su apreciada ayuda.

CORSINI, G.; FUENTES, R.; BUSTOS, L.; BORIE, E.; NAVARRETE, A.; NAVARRETE, D. \& FULGERI, B. temporomandibular disorders aigns and symtoms determination of 13 to 18 years old students from a school in Temuco, Chile. Int. J. Morphol., 23(4):345-352, 2005.

SUMMARY: Temporomandibular disorders (TMD) has been descrited as "Collective terms that involucre several clinical problems that compromise masticatory musculature, joints and associated structures". Epidemiologic studies have reported that there are common in children and adolescents. Showing prevalences ranging from $6 \%$ to $68 \%$ and $75 \%$ of adult population show some kind of functional alteration. The purpose of this study was to determine the prevalence of TMD signs and symptoms non existent in this commune, stablish the design effect for grade to calculate sample size for populational study and to determine stability of the test.

Cross sectinal study with non probabilistic (by convenience) sample and informed consent, taken from 116 students from the Baptist School of Temuco, who answered a test inquiring about subjective symptoms and then they were clinically examinations looking for signs, like: alterations, limitations or desviations of mandibular motion; pain to joint and muscular palpation; noises and bruxism. Autoreport Test: $77.6 \%$ repot one or more symptoms, articular noise $37.9 \%$; to feel jaw rigid $23.1 \%$ and headache, nape and temple, $35.3 \%$ Clinically: $85.3 \%$ showed one or more TMD signs; $50 \%$ joint noise; $8.6 \%$ mouth opening alteration; $18.1 \%$ diminished lateral motion; $8.6 \%$ tenderness to joint palpation; $4.3 \%$ tenderness to palpation an temporalis muscleand $6 \%$ masseterus; 46:6\% showed any bruxism manifestation. Correlation between this signs with gender and age showed only significant differences for age with mouth opening and Bruxism $(\mathrm{p}<0.05)$

There is agreement between what was perceived by the adolescents and what was clinically obsrved in maximun opening, chewing difficulty, noise and tendernees to joint palpation. In the analysis of the test and retest, great correlations was found between the answers. No statistically significant differences were found between signs and symptoms with gender. Desing effect: 1.23 . 


\section{REFERENCIAS BIBLIOGRÁFICAS}

Barone, A.; Sbordone, L. \& Ramaglia, L. Craniomandibular disorders and orthodontic treatment need in children. $J$. of Rehabilitation, 24: 2-7, 1997.

Conti, P. C.; Ferreira, P. M.; Pegorano, L. F.; Conti, J. V. \& Salvador, M. C. A cross sectional study of prevalence and etiology of signs and symptoms of temporomandibular disorders in high school and university student. J. Oralfacial Pain., 10:254-6, 1996.

DeBont, L. G.; Digkraaf, L. C. \& Stegenga, B. Epidemiology and natural progression of articular temporomandibular disorders. J. Oral. Surg. Oral Med. Oral Pathol. Oral Radiol and Endod., 83(1):72-6, 1997.

Dimitroulis, G. Temporomandibular disorders: A clinical update. British Medical J., 317(7152):190-4, 1998.

Egermark-Eriksson I.; Carlsson G. \& Magnusson T. A long term epidemiologic study of the relation ship between oclusal factors and mandibular dysfunction in children and adolescents. J. Dent Rest., 66(1):67-71, 1997.

Goldstein, B. H. TMD: A review of current understanding J.Oral Surg, Oral Med. Oral Pathol. Oral Radiol. and Endod., 88(4):379-85, 1999.

Greene, C. S. Etiology of temporomandibular disorders. Semin. Orthod., 14(4):222-8, 1995.

Kindernecht, K. E. Desórdenes Temporomandibulares: Un método práctico de selección. J. of Orthodopedics and Orthodontic Practice, 2(4):71-7, 1997.

Könönen, M.; Waltimo, A. \& Nystrom, M. Does clicking in adolescence less to painful TMJ loocking?. British Medical J., 4:1080-1, 1996

Krogstad, B. S.; Dahl, B. L.; Echersberg, T. \& Ogaard, B. Sex differences in sign aand symptoms from masticatory and other muscles in 19 years old individuals. J. Oral Rehabilitation, 19:435-40, 1992.

Kuttila, M.; Niemi, P.; Kuttila, S.; Alanem, P. \& LeBell, Y. TMD treatment need in relation to age, gender, stress and diagnostic subgroup. J. of Oralfacial Pain, 12:66-74, 1998.

Le Reche, L. Epidemiology of temporomandibular disorders; Implication for the investigations of etiologic factors. Crit. Rev, Oral Biol. Med., 8(3):291-305, 1997.

List, T.; Wahlum, K.; Wenneberg, B. \& Dworkin S. TMD in children and adolescents prevalence of Pain Gender differences, and perceived treatment need. J. Orofacial Pain, 13:9-20, 1999.
Magnusson, T.; Egermark-Eriksson, I. \& Carlsson, G. Four years longitudinal study of mandibular dysfunction in children. Community Dent. Oral Epidemiol., 13:117-20, 1985.

McNeill, C. History and evolution of TMD concepts. J. Oral Surg. Oral Med. Oral Pathol. Oral Radiol. and Endod., 83(1):51-60, 1997.

Okeson, J. Tratamiento de oclusión y afecciones temporomandibulares. 5. ed. Madrid, Elsevier, 2003.

McNeill, C. Temporomandibular Disorders, Guidelines for Classification, Assessment, and Management. 2. ed. Quintessence Publishimg Co., Illinois, 1993.

Salonen, L.; Hellden, L. \& Carlsson, G. Prevalence of signs and symptoms of dysfunction in the masticatory systems: An epidemiologic study in an adult Swedish population. $J$. Craniomandibular disord. Facial Oral Pain, 4:241-50, 1990.

Thurk, D. Psichosocial and behavoiral assessment of patiens with temporomandibular disorders. J. Oral Surg. Oral Med. Oral Pathol. Oral Radiol. and Endod., 83(1): 87-90, 1998.

Wänman, A. \& Ageberg, G. Mandibular dysfunction in adolescents. II Prevalence of signs. Acta Odontol Scand, 44:55-62, 1986.

Wahlund, K.; List, T. \& Dworkin, S. Temporomandibular disorders in children and adolescents: Reliability of a Questionnaire, clinical examination, and diagnostics. J. OroFacial Pain, 12:4251, 1998.

Wijer deA.; Lobbezoo-Scholte, A.M.; Steenks, M.H.\& Bosman, F. Reliability of clinical finding in temporomandibular disorders. J. Orofacial Pain, 9:181-91, 1995.

Dirección para correspondencia:

Dra. Gilda Corsini Muñoz

Departamento Odontología Integral

Facultad de Medicina

Universidad de La Frontera

Casilla 54-D

Temuco,

CHILE

E-mail: gcorsini@ufo.cl 